\title{
Multiple Constraints Consideration in Power System State Estimation
}

\author{
Jyoti. S. Bhonsle ${ }^{1}$, Dr. Anjali. S. Junghare ${ }^{2}$ \\ ${ }^{I}$ Electrical Engineering Department, Priyadarshini Institute of Engineering \& Technology, Nagpur, India \\ ${ }^{2}$ Electrical Engineering Department, Visvesvaraya National Institute of Technology, Nagpur, India
}

\begin{abstract}
State estimator is a likehood estimation system determines the best states of the power system. State estimation plays an essential role in modern Energy Management systems (EMS). This paper discusses a novel algorithm developed to detect any missing measurement data, bad data, elimination of bad data and status of network observability. The paper focus and considers the uncertainties in the available measurement data set while estimating the states of power system. The algorithm developed is simple and efficient. It is tested and implemented on IEEE 14 bus system.
\end{abstract}

Keywords - Bad data detection, Measurement Uncertainty, Observability, State Estimation, Weighted Least Square (WLS).

\section{Introduction}

State Estimation plays an essential role in modern Energy Management systems (EMS). Power system state estimation is the process carried out in the energy control centers in order to provide a best estimate of the system state based on the real-time system measurements and a pre-determined system model. A redundant set of real-time measurements, including bus voltage magnitudes, real and reactive power injections at the buses, real and reactive line power flows, and sometimes line current magnitudes, are collected from the entire network through the Supervisory Control and Data Acquisition (SCADA) system using Synchronized Phase Measurement Unit (PMU). These telemetered raw measurements are usually corrupted by different kinds of errors.

In this paper a novel algorithm is developed that identify and eliminate the bad data and provides missing data statistically to determine the best states of the power system. Multiple constraints are considered for estimating the best states of power system using Weighted Least Square (WLS) method. This paper focus and considers the uncertainty of the measured data from the latest developed and widely used measuring instrument i.e PMU (phasor measurement unit) for state estimation.

The paper is organized as follows. Section II introduces the widely used synchronized phase measurement unit, Section III deals with the various issues related to state estimation, Section IV introduces the formulation of state estimation solution using WLS method, in Section V, algorithm for bad data detection and elimination is developed and Section VI discusses the result and conclusion drawn from the analysis for IEEE 14 bus system.

\section{Synchronized Phase Measurement Unit}

Synchronized PMU is a monitoring device, which use synchronization signals from the global positioning system (GPS) satellites and provide the phasors of voltage and currents measured at a given substation. PMU has enough channels to record the bus voltage phasor at its associated bus and current phasors along all branches that are incident to this bus. It is a digital device which can process many measurement channels, so the possibility to install one unit for performing more than the one set of the measurements exists in all three phases. An additional benefit is that the measurements will contain a similar error regarding the accuracy class of the device, which for a PMU is typically lower than $1 \%$ of the total vector error (TVE). There is the advantage of possibly reducing the amount of the measurement devices in the network, for instance according to [23] PMU is capable of providing 7 phasors - 1 voltages and 6 currents (each phasor represents positive sequence calculated form 3 phases), but the security requirements need to be met.As the PMUs become more and more affordable, their utilization will increase not only for substation applications but also at the control centers for the EMS applications. PMU are placed at a strategic location to make the power system observable at set observability. The reliable communication infrastructure connecting the PMU with the phasor data concentrator (PDC), which collects all of the PMU measurements and can also be used as data source for Supervisory Control and Data Acquisition (SCADA) systems, should be provided to secure the consistency of the measurements. This issue should have a high priority if the measurement data is a critical measurement, meaning that if that specific data were missing, it would not be possible to perform the state estimation. 


\section{ISSUES RELATED To STATE ESTIMATION}

Usually the following assumptions are made in formulating the conventional static power system state estimation problem.

1) The 3-phase power system network is balanced, hence a 1-phase equivalent circuit can represent the system.

2) The system is operating in the steady state.

3) The parameters of the network are known and accurate.

4) The real-time topology of the network is precisely known.

5) All measurements are collected at the same instant and represent a true snapshot of the system state.

6) Measurement errors are independent random variables with zero mean Gaussian distribution.

7) Variances $\left(\sigma^{2}\right)$ of the measurement errors are exactly known.

In a real power system, some of these assumptions may not always hold, which will result in erroneous estimates. The difficulties of solving this kind of problems give rise to the so-called Generalized State Estimation algorithm.

One of the assumptions of the conventional state estimation is that measurement errors are independent random variables with zero mean Gaussian distribution. The telemetered measurements used in power system state estimation are final products of a chain of instruments, including instrument transformer, transducer, A/D (D/A) convertor, etc. Hence the uncertainties in the measurements are due to combination of random and systematic errors caused by those instruments. The raw measurements need to be calibrated before used by state estimator to satisfy the "zero mean error" requirement [3,13,14,15].

The conventional state estimation assumes the variances of the random errors are known and the measurements will be weighted by the inverses of random error variances to suppress their influences. The accuracy of the estimate results is greatly influenced by the weight vector. However, the random errors of measurements presented in control system are the combination of different sources of random errors, which come from different devices throughout the processing procedure. It is very difficult to precisely determine the variances of these random errors in advance. Moreover, some of the random error sources, such as those come from communication process, may be influenced by some real-time factors. This results in the time dependent property of variances. In this case, state estimation should be capable of setting measurement weight adaptively. The estimation and the auto-tuning of the variances of the measurements are necessary.

Observability analysis is another important procedure closely related to state estimation. Sometimes state estimation is not possible if it is not given enough measurements. If all the state variables (bus voltage magnitudes and relative phase angles) can be estimated using the available measurements, a system is said to be observable [20]. Accordingly, PMU are placed at a strategic location to make the power system completely observable at set observability even under contingency.

When a state estimation model fails to yield estimates within a degree of accuracy compatible with the standard deviations of the quantities estimated, one must conclude either that the measured quantities contain spurious data or that the model is unfit to explain the measured quantities. The procedure to identify and solve the former problem is called bad data analysis while for the later one is topology error detection/identification. There exist many bad data analysis techniques and they are successfully utilized. However, the conventional state estimators are still vulnerable to errors in the topology of the system, which show up when the assumed status of the circuit breakers and switches do not coincide with their true statuses [20,21].

Various methods for state estimation have been introduced in the past decades. Among those methods, Weighted Least Squares (WLS) algorithm is the most popular one. The objective function to be minimized of this method is chosen as the weighted sum of squares of the measurement residuals. Since this kind of problem can be solved by efficient numerical techniques, state estimators based on WLS approach have been installed in almost all the EMS systems all over the world.

\section{Formulation Of State ESTIMATION}

The problem of state estimation is usually formulated as a weighted least squares (WLS) problem, in which the objective function to be minimized is chosen as the weighted sum of squares of the measurement residuals. The measurement equation for a system modeled at the bus/branch level will take the following form:

$$
\mathrm{z}=\mathrm{h}(\mathrm{x})+\mathrm{e}
$$

where,

$\mathrm{z}$ is the measurement vector of dimension $\mathrm{m}$;

$\mathrm{h}(\mathrm{x})$ is the nonlinear relating the error free to the system states;

$\mathrm{x}$ is the state vector of dimension $\mathrm{n}$;

$\mathrm{e}$ is the measurement noise vector;

$\mathrm{n}, \mathrm{m}$ : the number of the state variables and measurement respectively.

Substituting the first order Taylor expansion of $\mathrm{h}(\mathrm{x})$ around some $\mathrm{x}_{0}$ in equation (1), we will have 
where,

$$
\Delta \mathrm{z}=\mathrm{H} \cdot \Delta \mathrm{x}=\mathrm{e}
$$

$$
\begin{aligned}
& \Delta \mathrm{z}=\mathrm{z}-\mathrm{h}\left(\mathrm{x}_{0}\right) \\
& \mathrm{H}=\frac{\partial h}{\partial x} \text { at } \mathrm{x}_{0} \\
& \Delta \mathrm{x}=\mathrm{x}-\mathrm{x}_{0}
\end{aligned}
$$

The weighted least square (WLS) estimate for $\mathrm{x}$ can be found by minimizing the following objective function:

$$
\mathrm{J}(\mathrm{x})=\sum_{i=1}^{m} \omega i(z i-h i(x))
$$

With the first order of Taylor expansion of $\mathrm{h}(\mathrm{x})$ shown in equation (2), the following equation will be solved iteratively to find the solution minimizing equation (3).

$$
\frac{\partial J(x)}{\partial x}=H^{s} \cdot W \cdot H \cdot \Delta x(k)-H^{s} \cdot W \cdot \Delta z(k)=0
$$

where, $W$ is the diagonal weight matrix.

Equation (4) can be rewritten as:

$$
\Delta \mathrm{x}(\mathrm{k})=\mathrm{G}^{-1} \cdot \mathrm{H}^{\prime} \cdot \mathrm{W} \cdot \Delta \mathrm{x}(\mathrm{k})=0
$$

where, $G=\square \mathrm{H}^{\prime} \cdot \mathrm{W} \cdot \mathrm{H} \quad$ is called the gain matrix.

The WLS estimation problem given by equation (3) and equation (5) can be solved iteratively until $|\Delta \mathrm{x}(\mathrm{k})|$ become smaller than a threshold.

\section{BAD DATA DETECTION / IDENTIFICATION ALGORITHM}

When a state estimation program fails to yield accurate estimates, it is either due to the erroneous measurements i,e bad data problem or due to a modeling error i.e topology error problem or both.

A common technique used for bad data processing is the normalized residuals test ( $\mathrm{r}^{\mathrm{n}}$ test). The $\mathrm{r}^{\mathrm{n}}$ test used in WLS method is described below:

Assume that the state estimate $\mathrm{x}^{\wedge}$ has already been computed from equation (5). The residuals of the measurements are defined as:

$$
\mathrm{r}^{\wedge}=\mathrm{z}-\mathrm{h}\left(\mathrm{x}^{\wedge}\right)
$$

The relationship between the residuals and the measurement errors can be obtained as:

where,

$$
\mathrm{r}^{\wedge}=\mathrm{S} \cdot \mathrm{e}
$$

$$
\mathrm{S}=\mathrm{I}-\mathrm{H} \cdot \mathrm{G}^{-1} \mathrm{H}^{\prime} \cdot \mathrm{W}
$$

Then the covariance matrix of the residuals can be computed by:

$$
\mathrm{Rr}^{\wedge}=\mathrm{S} \cdot \mathrm{R}_{\mathrm{z}} \cdot \mathrm{S}
$$

The normalized residuals can be obtained as:

$$
r_{n}=\left(\operatorname{diag}\left(\operatorname{Rr}^{\wedge}\right)\right)^{-1 / 2} \cdot r^{\wedge}
$$

If there exist some normalized residuals greater than the probability threshold, bad data will be detected in the measurement set. Further more, the measurement with the largest $r^{n}$ will be identified as bad data in most of the cases.

Steps to developed algorithm :

1. Run load flow for the network

2. Obtained true values of system states

3. Run WLS algorithm

4. Obtained best estimated values of system states

5. Obtained Residuals

6. Consider the measurement uncertainty and repeat the above steps

7. Generate the measurement set with bad data

8. Check the threshold for bad data

9. Identify and Eliminate the bad data from the set

10. Generate the measurement set with reduced system state

11. Check the observability of system state

12. Check the missing data in measurement set

13. Estimate the missing data

14. Check for bad data, missing data and system observability considering measurement uncertainty for the given measurement set

15. Obtained the best system states. 


\section{RESULT AND CONCLUSION}

State Estimation using WLS incorporated with bad data detection algorithm is performed on standard IEEE 14- Bus system. Two data set are considered for analysis, one with specific measurement and other with certain degree of uncertainties. Uncertainty in measurement of data considered is with maximum uncertainties in the measurement of voltage, current and phase angle as $0.02 \%, 0.03 \%$ and 0.01 degree respectively.

Observability of power system is checked for various sets of provided measurement. A data set for insufficient data is also provided for the testing and observability checking purpose. Bad data detection algorithm is successfully incorporated in state estimation formulation. Fig. 1 shows the IEEE 14 bus system.

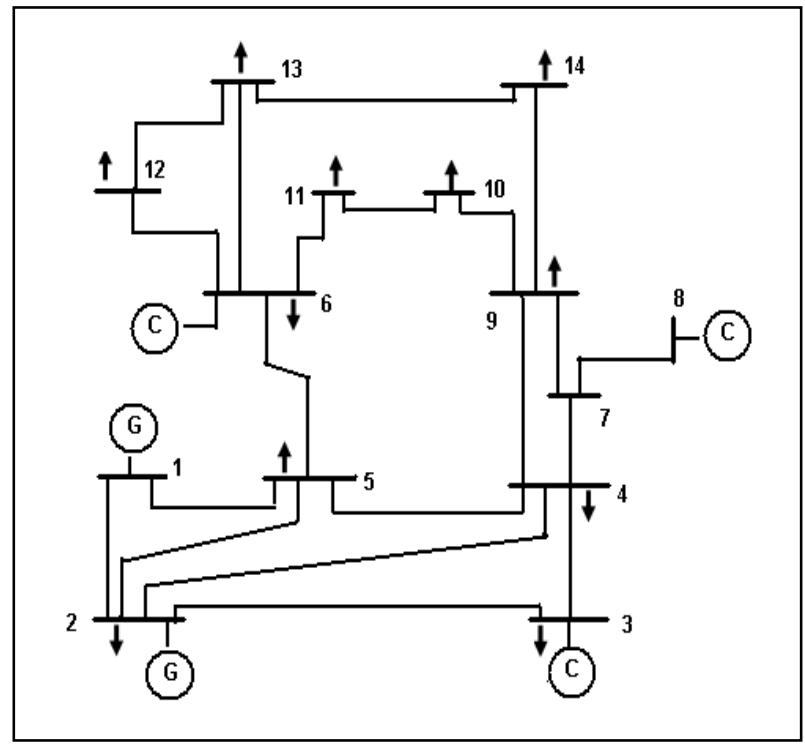

Figure 1 IEEE 14 bus system

Fig. 2 and Fig. 3 graphical shows the true values and estimated values of voltage magnitude and phase angle for IEEE 14 bus system respectively.

Table I gives the optimum number of PMU with their location for IEEE 14 bus system under different consideration for one-depth of unobservability. Fig. 4 and Fig. 5 graphical shows estimated values of voltage magnitude and angle with upper and lower limits respectively considering the maximum uncertainties for IEEE 14 bus system respectively. Table II shows the index and value of performance index in each iteration for bad data detection and elimination using novel algorithm developed incorporating WLS .

True and Measurement data set is generated for IEEE 14 bus system considering multiple constraints such as bad data, missing data, network observability and measurement uncertainties for testing purpose of developed algorithm. Providing reduced measurement data set leads to system unobservability.

Algorithm developed detects any missing measurement data and provide approximate data, detects and eliminates bad data and status of network observability such as observable or not observable without and with considering the maximum uncertainties in measurement data set.

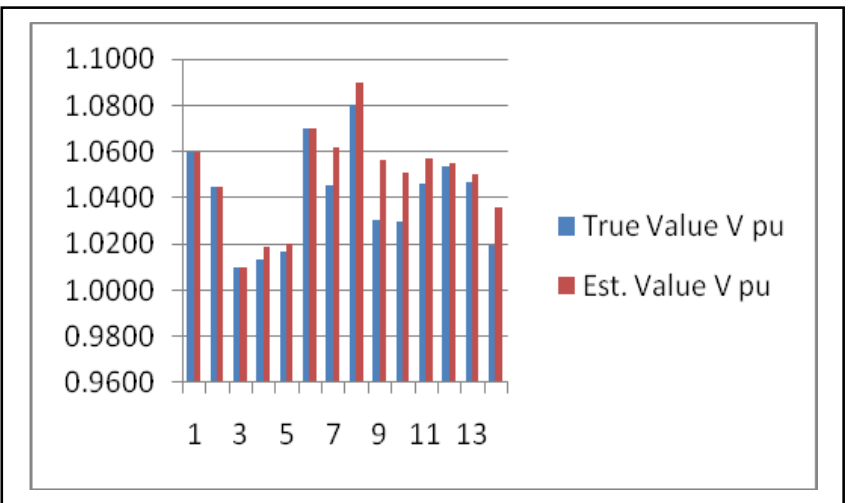

Figure 2 True value and Estimated values of voltage magnitude 


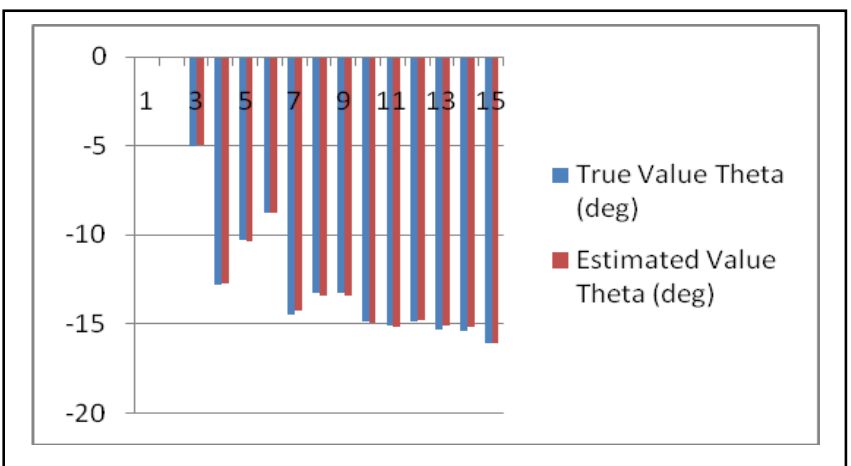

Figure 3 True value and Estimated values of phasor angle

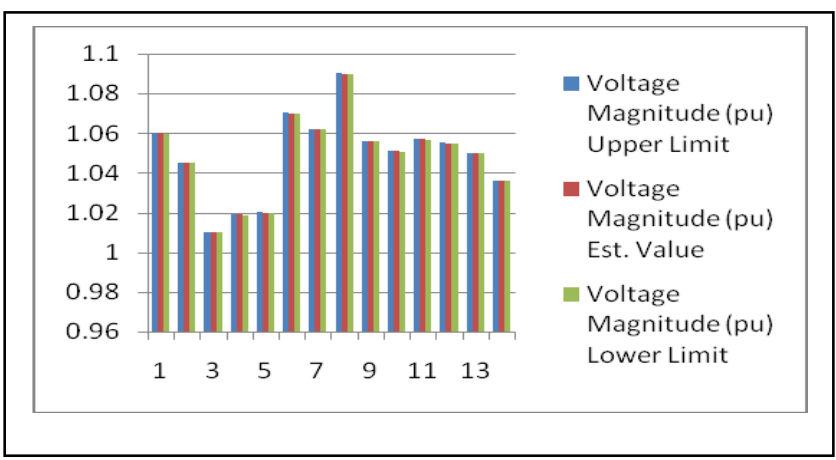

Figure 4 Estimated values of voltage magnitude with upper and lower limits

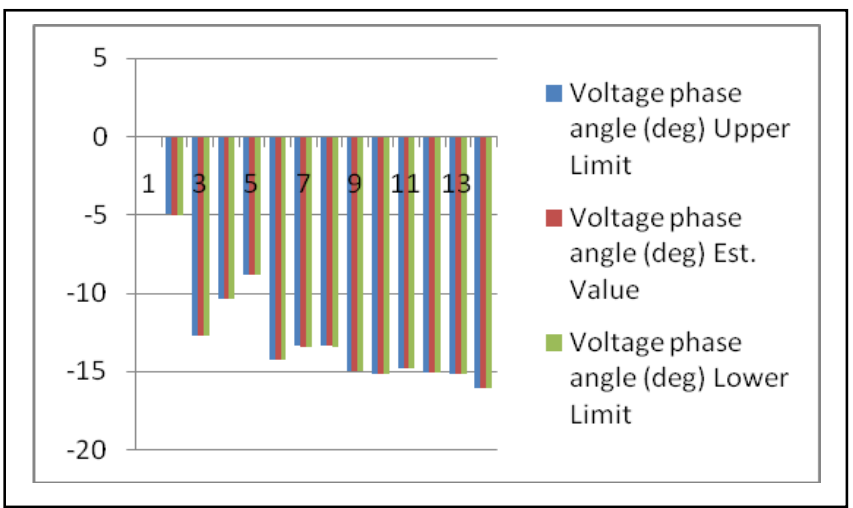

Figure 5 Estimated values of phasor angle with upper and lower limits

Table I (Only PMU based measurement for one-depth of UnObservability)

\begin{tabular}{|c|c|c|c|}
\hline System & Constraints & $\begin{array}{c}\text { Optimum } \\
\text { No. of PMU }\end{array}$ & Location of PMU \\
\hline \multirow{2}{*}{$\begin{array}{c}\text { IEEE } \\
\text { 14-bus } \\
\text { system }\end{array}$} & $\begin{array}{c}\text { w/o failure of PMU or } \\
\text { Communication line }\end{array}$ & 2 & 4,6 \\
\cline { 2 - 4 } & $\begin{array}{c}\text { With the failure of PMU or } \\
\text { Communication line }\end{array}$ & 4 & $4,5,6,9$ \\
\hline
\end{tabular}

Table IV Performance Index

\begin{tabular}{|c|c|c|}
\hline Iteration & Index & Performance Index \\
\hline 1 & 89 & 17.12 \\
\hline 2 & 110 & 24 \\
\hline 3 & 42 & 8.71 \\
\hline 4 & No more bad data & 4.94 \\
\hline
\end{tabular}




\section{References}

[1] G. Valverde, S. Chakrabarti, E. Kyriakides and V. Terzia, “ A Constrained Formulation for Hybrid State Estimation”, IEEE Transaction on Power Systems, 2010

[2] S. Chakrabarti, E. Kyriakides, G. Ledwich and A. Ghosh, “ Inclusion of PMU current phasor measurements in a power system state estimator", IET Gen. Transm. And Distrib. 2010, Vol. 4, Iss. 10, Pp. 1104-1115

[3] S. Chakrabarti and E. Kyriakides, "PMU measurement uncertainity consideration in WLS state estimation", IEEE Transaction Power Systems, Vol. 24, No. 2 May 2009 pp. 1062-1071

[4] Amit Kumar and S. Chakrabarti, "An ANN based Hybrid State Estimator", $16^{\text {th }}$ National Power Systems Conference, $15^{\text {th }}-17^{\text {th }}$ Dec, 2010, pp. 734-737

[5] R. Neela and P. Aravindhababu, "A new decoupling strategy for power system state estimation", Energy Conversion and Management, Vol.50, No. 8, pp. 2047-2051, 2009

[6] P. Aravindhababu and R. Neela, "A reliable and fast decoupled WLS state estimation for power systems", Electric Power Components and Systems, Vol. 36, No. 11, pp. 1200-1207, 2008

[7] T. S. Bi , X. H. Qin and Q. X. Yang, "A novel hybrid state estimator for inclusing synchronized phasor measurements", Electric Power Systems Research, Vol. 78, pp. 1343-1352, 2008

[8] Jun Zhu and A. Abur, “ Improvements in network parameter error identification via synchronized phasors", IEEE Transaction on Power Systems, Vol. 25, No. 1, Feb 2010

[9] Jian Ma, Yousu Chen, Zhenyu Huang and Pak Chung Wong, " Using state estimation resi duals to detect abnormal SCADA data", 978-1-4244-6547-7/10, IEEE, 2010

[10] Jun Zhu and A. Abur, “Bad data identification when using Phasor Measurements”, 978-1-4244-2190-9/07, IEEE, 2007, pp. 16761681

[11] M. Zhou, V.A. Centeno, J.S. Thorp and A.G. Phadke, “An alternative for including Phasor Measurements in State Estimators”, IEEE Transaction on Power Systems, Vol. 21, No. 4, pp. 1930-1937 Nov 2006

[12] Jun Zhu and A. Abur, "Effect of phasor measurements on the choice of reference bus for state estimation", Proceedings, IEEE PES General Meeting, $24^{\text {th }}-28^{\text {th }}$ June 2004

[13] S. Chakrabarti, D. Eliades, E. Kyriakides and M. Albu, “ Measurement uncertainty considerations in optimal sensor deployment for state estimation ", IEEE Symp. Intelligent Signal Processing, Oct 2007

[14] A.K. Al-Othman and M.R. Irving, "Uncertainty modeling in power system state estimation", Proc. Inst. Elect. Eng. Gen. Trans. Distri. Vol 152, No. 2, 2005, pp. 233-239

[15] A.K. Al-Othman and M.R. Irving, " A comparative study of two methods for uncertainty analysis in power system state estimation", IEEE Trans. Power Systems, Vol. 20, No. 2, 2005, pp. 1181-1182

[16] Dalu Li, Rui Li, Yuanzhang Sun and Han Chen, "WAMS/SCADA Hybrid system state estimation algorithm with double constraints", 978-1-4244-2487-5/09, IEEE, 2009

[17] Ni Xiaoping, Zhang Buhan, “New state estimation algorithm with equality constraints”, Automation of Electric Power Systems, 2001, Vol. 25, No.21, pp. 42-44

[18] Qin Xiaohui, Bi Tianshu, Yang Qixun, “A new method for hybrid nonlinear state estimation with PMU ”, Automation of Electric Power Systems, 2007, Vol. 31, No.04, pp. 28-32

[19] R.F.Nuqui and A. G. Phadke, “Hybrid Linear state estimation utilizing synchronized phasor measurements”, Proc. PowerTech July 2007

[20] J. Chen and A. Abur, “Enhanced Topology processing via Optimal measurement design”, IEEE Trans. On Power Systems, Vol. 23, No. 3, pp. 845-852, Aug 2008

[21] Jun Zhu and A. Abur, "Improvements in network parameter error identification via synchronized phasors", IEEE Trans. On Power Systems, Vol. 25, No.1, 2010, pp.44-50

[22] Ali Abur and A. Gomez-Exposite, " Detecting Multiple solutions in state estimation in the presence of current magnitude measurements", IEEE Trans. On Power Systems, Vol. 12 (1), pp. 370-375, Feb 1997

[23] J.S.Bhonsle and A.S.Junghare, "A Novel Approach for the Optimal PMU placement using binary integer programming technique”,(IJEEE) ISSN (Print) 2231 - 5284, Vol-1, Iss-3, 2012, pp. no. 67-72 